\title{
Assessing Postconcussive Reaction Time Using Transport-Based Morphometry of Diffusion Tensor Images
}

\author{
(D). Kundu, (D)A. Ghodadra, (D)S. Fakhran, (D).M. Alhilali, and (D) G.K. Rohde
} O- $\equiv$

\begin{abstract}
BACKGROUND AND PURPOSE: Cognitive deficits are among the most commonly reported post-concussive symptoms, yet the underlying microstructural injury is poorly understood. Our aim was to discover white matter injury underlying reaction time in mild traumatic brain injury DTI by applying transport-based morphometry.
\end{abstract}

MATERIALS AND METHODS: In this retrospective study, we performed DTI on 64 postconcussive patients (10-28 years of age; $69 \%$ male, 31\% female) between January 2006 and March 2013. We measured the reaction time percentile by using Immediate Post-Concussion Assessment and Cognitive Testing. Using the 3D transport-based morphometry technique we developed, we mined fractional anisotropy maps to extract the common microstructural injury associated with reaction time percentile in an automated manner. Permutation testing established statistical significance of the extracted injuries. We visualized the physical substrate responsible for reaction time through inverse transport-based morphometry transformation.

RESULTS: The direction in the transport space most correlated with reaction time was significant after correcting for covariates of age, sex, and time from injury (Pearson $r=0.44, P<.01$ ). Inverting the computed direction using transport-based morphometry illustrates physical shifts in fractional anisotropy in the corpus callosum (increase) and within the optic radiations, corticospinal tracts, and anterior thalamic radiations (decrease) with declining reaction time. The observed shifts are consistent with biologic pathways underlying the visual-spatial interpretation and response-selection aspects of reaction time.

CONCLUSIONS: Transport-based morphometry discovers complex white matter injury underlying postconcussive reaction time in an automated manner. The potential influences of edema and axonal loss are visualized in the visual-spatial interpretation and responseselection pathways. Transport-based morphometry can bridge the gap between brain microstructure and function in diseases in which the structural basis is unknown.

ABBREVIATIONS: $\mathrm{FA}=$ fractional anisotropy; ImPACT = Immediate Post-Concussion Assessment and Cognitive Testing; mTBI = mild traumatic brain injury; $\mathrm{TBM}=$ transport-based morphometry

C

ognitive deficits are among the most commonly reported symptoms after mild traumatic brain injury (mTBI). ${ }^{1-6}$ Both transient and persistent deficits in processing speed, attention, and working memory are associated with mTBI. ${ }^{7}$ Furthermore, persistent cognitive deficits may accelerate aging-related cognitive decline. ${ }^{2}$ Yet, despite its ubiquity, the microstructure under- lying postconcussive cognitive deficit is difficult to assess. Routine CT and MR imaging findings are often negative. Although diffusion tensor imaging can capture diffuse white matter injury, ${ }^{8}$ mTBI is still diagnosed clinically because damage is subtle and spatially diffuse. ${ }^{7,9}$ The microstructural injury is challenging to
Received December 26, 2018; accepted after revision April 27, 2019. From the Department of Biomedical Engineering, Electrical and Computer Engineering (G.K.R.), University of Virginia, Charlottesville, Virginia; Department of Biomedical Engineering at Carnegie Mellon University and Medical Scientist Training Program (S.K.), University of Pittsburgh Medical Center, Pittsburgh, Pennsylvania; Department of Radiology (A.G.), Banner Health and Hospital Systems, Mesa, Arizona; and Department of Neuroradiology (S.F.), Barrow Neurological Institute, Phoenix, Arizona. Dr Alhilali is currently at the Department of Internal Medicine, University of Pittsburgh Medical Center, Pittsburgh, Pennsylvania.

This work was supported, in part, by the National Science Foundation award CCF 1421502 and National Institutes of Health awards GM130825 and GM090033.
A subset of these results was presented at: Radiological Society of North America's Scientific Assembly and Annual Meeting: "Transport-Based Morphometry of Diffusion Tensor Images for Assessment of Postconcussive Reaction Time." November 27 to December 2, 2016; Chicago, Illinois.

Please address correspondence to Shinjini Kundu, MD, PhD, Medical Scientist Training Program, University of Pittsburgh/Carnegie-Mellon University, 526 Scaife Hall, 3550 Terrace St, Pittsburgh, PA 15261; e-mail: shk71@pitt.edu; @ShinjiniKundu

- Indicates open access to non-subscribers at www.ajnr.org

$\equiv$ Indicates article with supplemental on-line appendix.

Indicates article with supplemental on-line photos.

http://dx.doi.org/10.3174/ajnr.A6087 
distinguish from a background of normal anatomic variability and accumulated insults with time. ${ }^{10}$

Given its complexity, traditional analysis has permitted only a limited view of the microstructural perturbations. Conventional methods have sought to index injuries to individual tracts (ie, ROI analysis; Tract-Based Spatial Statistics, TBSS; http://fsl.fmrib.ox.ac. $\mathrm{uk} / \mathrm{fsl} / \mathrm{fslwiki} / \mathrm{TBSS}$; and so forth) or individual voxels (ie, voxelbased analysis, potholes and molehills analysis, and so forth). ${ }^{7,10,11}$ However, techniques treating each voxel or ROI as an independent variable cannot provide adequate insight into the interconnectedness of injuries across multiple regions as a function of cognition. ${ }^{6,7}$ Furthermore, visual inspection is particularly insensitive to subtle- butinterconnected biophysical shifts in tissue architecture. ${ }^{12}$

In our prior work, we developed 3D transport-based morphometry (TBM) as a technique to assess complex physical changes in brain tissue that may be undetectable by visual inspection. ${ }^{13}$ By computing the effort needed to morph the brain tissue distribution in one image into another, TBM statistically mapped joint interaction across multiple brain regions. ${ }^{13}$ Unlike traditional approaches, TBM could explain the relevant microstructural shifts underlying the clinical assessment visually because it is a generative technique. ${ }^{13}$ Furthermore, TBM can discover the common substrate underlying reaction time in a fully automated manner.,

This study investigated the brain microstructure associated with postconcussive reaction time, one of the most frequently reported cognitive deficits after mTBI. ${ }^{1,3.4,14,15}$ Given new interventions such as exercise rehabilitation, ${ }^{16,17}$ it is crucial to identify objective substrates underlying postconcussive cognition to assess progress in treatment and understand repair mechanisms. Particularly, studying the acute and postacute phases of recovery could reveal new targets for early treatment monitoring. TBM has never before been applied to investigate white matter injury associated with postconcussive cognition. Given the known limitations of traditional techniques, we hypothesize that if a common structural substrate underlying postconcussive reaction time exists, it can be uncovered using TBM.

\section{MATERIALS AND METHODS Subject Cohort}

In this retrospective study, institutional review board approval was obtained, and informed consent was waived. The electronic medical record was searched for DTI studies performed for mTBI between January 1, 2006, and March 1, 2013, at the University of Pittsburgh Medical Center. Studies were identified using keywords "concussion," "mild traumatic brain injury," and "diffusion tensor imaging." Inclusion criteria were the following: 10-50 years of age, witnessed closed head trauma, no focal neurologic deficit, loss of consciousness of $<1$ minute, posttraumatic amnesia of $<30$ minutes, English language proficiency, and available neurocognitive testing with Immediate Post-Concussion Assessment and Cognitive Testing (ImPACT; https://scipol.duke.edu/track/immediate-post-concussionassessment-and-cognitive-testing-impact). Exclusion criteria were the following: prior neuropsychiatric illness (2 patients), abnormal CT or conventional brain MR imaging findings including large focal abnormalities such as encephalomalacia not attributable to current trauma (3 patients), history of substance abuse (3 patients), lack of DTI (4 patients), lack of neurocognitive assessment (6 patients), total symptom score of zero (3 patients), and
Table 1: Demographic information and causes of injury

\begin{tabular}{lc}
\hline \multicolumn{1}{c}{ Participant Characteristics } & $\begin{array}{c}\text { Number of } \\
\text { Participants }\end{array}$ \\
\hline Demographics & \\
Age, time of imaging (range) (mean) (yr) & $10-28(16.1 \pm 2.9)$ \\
Male participants (\%) & $44(68.8)$ \\
Time from injury to imaging (mean) (days) & $55 \pm 91$ \\
Causes of mTBI & \\
Sports injury (\%) & $42(65.6 \%)$ \\
Motor vehicle collision (\%) & $6(9.4 \%)$ \\
Bicycle accident (\%) & $2(3.1 \%)$ \\
Fell down stairs (\%) & $2(3.1 \%)$ \\
Fell off horse (\%) & $2(3.1 \%)$ \\
Other $\left.{ }^{\text {a }} \%\right)$ & $10(15.6 \%)$ \\
\hline
\end{tabular}

${ }^{a}$ Various civilian injuries.

inability to align fractional anisotropy (FA) images (2 patients). The cohort included patients in the immediate $(n=13)$, acute $(n=30)$, subacute $(n=10)$, and chronic $(n=11)$ phases of injury (acute, 1-6 weeks; subacute, $7-12$ weeks; chronic, $>12$ weeks). ${ }^{18}$ The final cohort contained 64 subjects. A prior article ${ }^{11}$ used this cohort to examine postconcussive headache. Demographic information and causes of injury are summarized in Table 1. Unlike voxel-based analysis or ROI techniques used in prior studies, we evaluated postconcussive reaction time using the TBM technique to enable both discovery and visualization of novel structural substrate.

A neuropsychological examination was performed by a single neuropsychologist with $>15$ years of experience in treating patients with mTBI. Reaction time was determined as part of the ImPACT. ${ }^{19}$ Although ImPACT included other cognitive measures, this study focused on reaction time, one of the most common postconcussive cognitive sequelae ${ }^{20}$ and a sensitive marker of recovery following concussion. ${ }^{20,21}$ ImPACT reaction time for each subject is a percentile based on normative data from baseline testing of $>17,000$ athletes as part of presport participation. Percentile information accounts for both sex and age.

\section{MR Imaging Acquisition and Preprocessing}

All subjects underwent an identical imaging protocol on the same system during the imaging time period. DTI was performed with a 1.5T unit (Signa; GE Healthcare, Milwaukee, Wisconsin) and a standard head coil. A single-shot echo-planar sequence was used (TR = $4000 \mathrm{~ms}, \mathrm{TE}=80 \mathrm{~ms}, \mathrm{NEX}=2$, section thickness $=5 \mathrm{~mm}$, matrix $=$ $128 \times 128, \mathrm{FOV}=260 \mathrm{~mm})$. Diffusion gradients were set in 25 noncollinear directions using $2 \mathrm{~b}$-values $\left(b=0\right.$ and $1000 / \mathrm{mm}^{2}$ ).

FA maps were generated to characterize white matter integrity using the Brain Diffusion Toolbox, ${ }^{22,23}$ which is part of the DTI pipeline of FSL. ${ }^{24,25}$ The FA maps included both gray and white matter and were registered to the Montreal Neurological Institute atlas by a 12-parameter affine transformation. A common reference image was computed through the Euclidean average of all images across the study population.

\section{Analyzing FA Spatial Distribution Using Transport-Based Morphometry}

The microstructure differentiating high and low reaction time was not perceptible visually as demonstrated in Fig 1, motivating the use of $\mathrm{TBM}^{13,26-28}$ to discover the complex pattern. Our 3DTBM technique ${ }^{13}$ is an image-transformation framework that fa- 


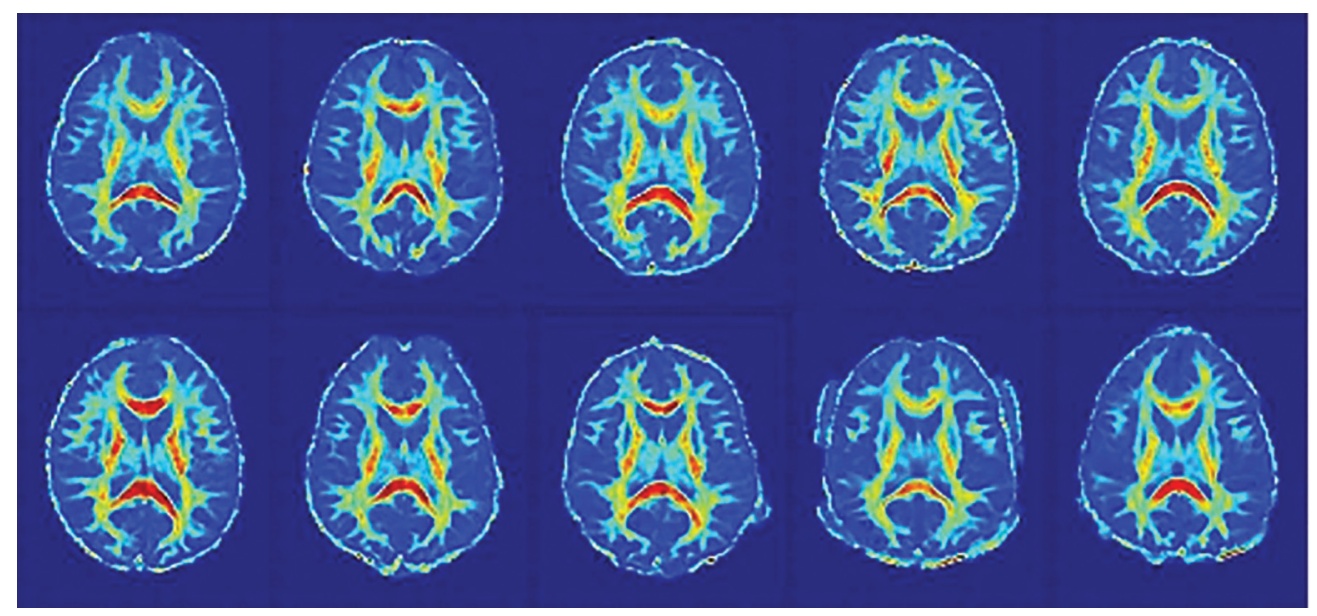

\section{Best reaction times}

\section{Worst reaction times}

FIG 1. FA maps of 10 different postconcussive patients. The same axial slice is shown for patients with the best reaction time percentiles (upper row) and worst reaction time percentiles (lower row). These images illustrate the challenge that FA maps corresponding to the best and worst reaction times are not easily differentiated by visual inspection because the abnormal variations related to reaction time cannot be discerned from the other heterogeneous variations. Intelligent computer-aided techniques are needed to differentiate these images.

cilitates the discovery of this pattern through analysis in a transform domain. ${ }^{26-28}$ On-line Fig 1 illustrates the system diagram in the On-line Appendix. The interested reader is referred to Kundu et $\mathrm{al}^{13}$ for the mathematic derivation of 3D-TBM. We provide only a brief description here.

The similarity in the FA spatial distribution with respect to a reference is quantified in a transport map for each subject: a vector field morphing an individual FA image into the common reference. The optimal transport map minimizes FA transport to match the common reference. On-line Fig 2 shows FA maps and corresponding transport maps for 4 sample subjects.

The optimal transport map is unique for a pair of images, ${ }^{13}$ ensuring a bijective transformation. Equations A1 and A2 describe analysis and synthesis equations for forward and inverse transforms, respectively, in the On-line Appendix. Transformation is nonlinear; thus, complex patterns are more sparsely characterized in the transport domain and correspond to distances on the Riemannian manifold. Computing Riemannian distances with respect to a common template allows linearization of the optimal mass transport metric and reduces the number of operations from $N(N-1) / 2$ to $N$. Prior work shows that pattern discrimination using the linear optimal transport distance is enabled when the template lies sufficiently close to the Riemannian manifold. ${ }^{27,28}$ Because the TBM transformation is bijective, we can generate de novo images along the regression direction in transport space through inverse transformation. The latter is enabled because TBM is generative. The ability to ultimately visualize the complex shift in microstructural profile initially imperceptible to visual inspection (Fig 1) is a unique contribution of TBM. We implemented all 3D-TBM codes in Matlab (MathWorks, Natick, Massachusetts) as described in Kundu et al. ${ }^{13}$

\section{Assessing the Microstructural Relationship with Reaction \\ Time}

Statistical learning is performed in the transport domain to extract relevant image features. First, transport maps generated by the transformation are vectorized and concatenated into a standard data matrix. Then, the principal components analysis technique (Equation A3) is used to eliminate data dimensions con- tributing little to the variance in the dataset. The data matrix is projected onto the topmost $d$ eigenvectors associated with $90 \%$ of the variance in transport maps to create the reduced-dimension data matrix $X \in \mathbb{R}^{d \times n}(d \ll p)$. Let $\nu \in \mathbb{R}^{n \times 1}$ be the reaction time percentile mapped to squared values (to increase separation of lower from higher percentiles). The direction in transport space that results in the strongest linear correlation is computed with the reaction time percentile $\nu$ (Equation 1$)^{26}$ :

$$
w_{\text {corr }}=\arg \max _{w} \frac{w^{T} X \nu}{\sqrt{w^{T} w}}=\frac{X \nu}{\sqrt{\nu^{T} X^{T} X \nu}} .
$$

Here, $w_{\text {corr }}$ is a vector field synthesized in the transport domain that quantifies the direction and amount of FA redistribution most correlated with the reaction time percentile. Covariates are removed according to Equation 2.

$$
\nu=y-Z\left(Z^{T} Z\right)^{-1} Z^{T} y .
$$

Here, $\nu$ describes the residual from the reaction time percentile $\left(y \in \mathbb{R}^{n \times 1}\right.$ ) that is decorrelated and orthogonal to the $c$, confounding variables in $Z \in \mathbb{R}^{n \times c}$. This study corrects for age, sex, and time from injury as covariates. Statistical significance of the computed direction was assessed using permutation testing with $T=1000$ tests. The reported $P$ value is the fraction of times over $T$ tests that the Pearson correlation coefficient was higher when labels $v$ are randomly assigned to subject data than when computed based on the original assignment. We implemented all statistical analysis code in Matlab.

\section{RESULTS}

\section{Assessing the Microstructural Relationship with Reaction Time}

As illustrated in Fig 1, visual inspection does not elucidate the changes characteristic of poor postconcussive reaction time. The computed tissue distribution from the transport space was significantly associated with the reaction time percentile after correcting for covariates according to Equation 2 (Pearson $r=0.44, P<$ $.01)$. The strength of the association is moderate. Furthermore, the pattern of injury identified by TBM is specific to cognitive 
symptoms following mTBI because it is not significantly correlated with any other ImPACT measures except processing-speed percentile (Table 2).

On-line Fig 3 shows the scatterplot of subjects when their images are projected onto the most correlated direction computed in the transport space. The mean image $I_{0}(\mathrm{x})$ maps to a projection score of zero. Each point in the scatterplot represents a brain image in the dataset. According to the linear regression model constructed in the transport space, increasing reaction time percentile (faster reaction times) is associated with increasing projection scores, and decreasing reaction time percentile (slower reaction times) is associated with decreasing projection scores.

\section{Understanding Complex Correlations Visually}

In addition to confirming a correlation between the reaction time percentile and microstructure, projection scores can be interpreted clinically. Reverse TBM transformation can map any given projection score to visualize a computer-generated image. Figure 2 shows

Table 2: Correlation between TBM direction and other ImPACT measures

\begin{tabular}{lcc}
\hline \multicolumn{1}{c}{ ImPACT Measure } & $\begin{array}{c}\text { Pearson } \\
\text { Correlation }\end{array}$ & $\boldsymbol{P}$ \\
\hline Anxiety & -0.06 & .68 \\
Cervicalgia & -0.06 & .70 \\
Headaches & -0.18 & .91 \\
Total ImPACT score & -0.29 & .98 \\
Processing speed percentile & 0.31 & $<.01^{\mathrm{a}}$ \\
Sleep-wake disturbance & -0.24 & .97 \\
Time to recovery & -0.05 & .67 \\
Tinnitus & -0.05 & .61 \\
Verbal memory & 0.20 & .06 \\
Visual memory & 0.20 & .05 \\
\hline
\end{tabular}

a The discovered pattern of injury does not correlate with any other ImPACT measures except processing-speed percentile. visualizations of dynamic brain morphology corresponding to varying projection scores. The images are synthesized on the basis of the study population. Note that simple linear regression in transport space can characterize the nonlinear, spatially diffuse, white matter injury pattern in the image domain.

The projection scores across the horizontal axis in Fig 2 correspond to those in the horizontal axis of On-line Fig 3. The images shown are computer-generated by TBM from points sampled along the line of best fit where the projection score spans -2 to +2 SDs from the mean. The FA maps are colored to aid in visualization. The values represent relative density, a measure of FA concentration. Two axial slices are shown that best summarize the morphologic differences from a low-reaction-time percentile to a high-reaction-time percentile. The population mean image $I_{0}(\mathrm{x})$ is indicated by the projection score zero in Fig 2 . The images at the negative projection scores correlate most strongly with the lowreaction-time percentile, while the images at the positive projection scores correlate most strongly with high-reaction-time percentile (On-line Fig 3). Progressing from a low-to-high reaction time percentile, the statistical model illustrates that FA in the corpus callosum decreases while increasing in the optic radiations, corticospinal tract, and anterior thalamic tracts.

\section{Individual Assessment of White Matter Injury Associated with Reaction Time}

In addition to identifying common structural correlates of reaction time, TBM also identifies white matter injury in individual patients. The direction computed in transport space can clinically assess whether the patterns of white matter injury in Fig 2 are present in an individual patient. The transport map for an individual patient can be projected onto this direction to yield a projection score for the patient. The projection scores can be interpreted on the basis of the relationship with reaction time performance illustrated in On-line Fig 3 to evaluate whether cognitive deficits may be present. Figure 3 illustrates the FA map of a patient with a fast reaction time in the 94th percentile that maps to a high positive projection score and that of a patient with slow reaction time in the 21st percentile that maps to a low negative projection score, a potential metric by which to differentiate these patients. A key strength of TBM is its ability to assess spatially diffuse, complex white matter patterns that are not easily identified visually or by an ROI-based perspective.

Furthermore, Fig 3 shows that the transport map may reveal new numeric assessment tools. The determinant of the Jacobian computed from the transport maps measures the joint changes in FA concentration across multiple white matter tracts. An increase in FA concentration is indicated by a value of $>1$, and decreases are indicated by a value of $<1$. We see that the direction of FA change in multiple white matter tracts with respect

FIG 2. Most correlated direction. Images corresponding to the most correlated direction in transport space show decreasing FA in the corticospinal tracts, anterior thalamic radiations, and optic radiations with the low reaction time percentile corresponding to the scatterplot in On-line Fig 3. The FA in the corpus callosum increases as reaction time decreases. 


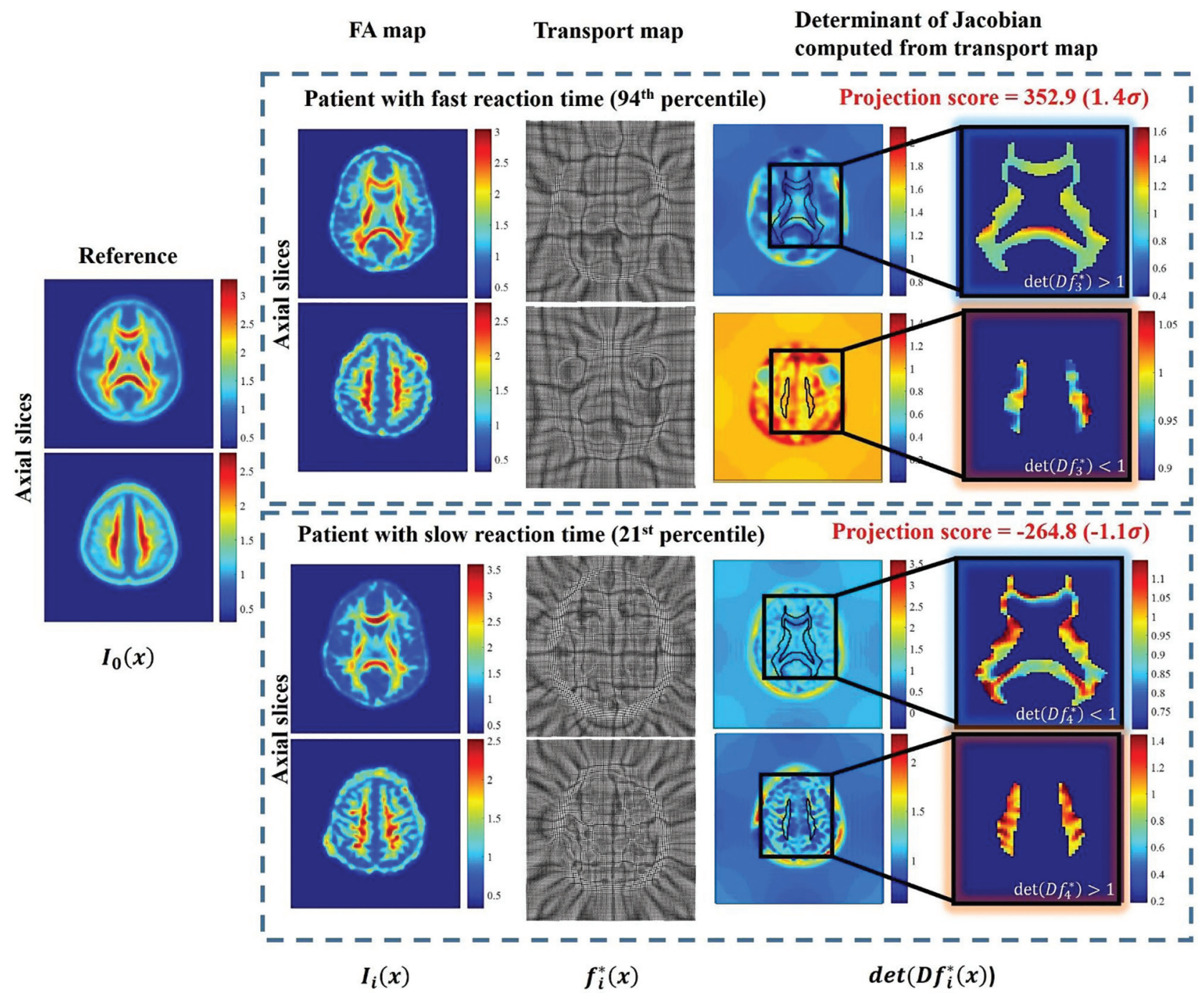

FIG 3. TBM can help assess cognitive deficits in individual patients using the computed direction to assess whether the complex and spatially distributed patterns of white matter injury identified by Fig 2 are present. When transport maps are projected onto this direction, a projection score is generated that can be used to differentiate patients according to the relationship in On-line Fig 3 . The subjects shown correspond to $i=3$ and 4 in Fig 1. The direction of FA change with respect to the reference in individual patients corroborates the patterns identified in Fig 2 .

to the common reference corroborates those identified by Fig 2 for a patient with high and low reaction times.

\section{DISCUSSION}

This article investigates the microstructure underlying postconcussive reaction time. Our prior work developing 3D-TBM demonstrated its ability to uncover microstructural circuits not adequately assessed through visual inspection or conventional methods. ${ }^{13}$ This article builds on prior work to propose a new framework to bridge microstructure and postconcussive cognitive assessment. We discovered an FA spatial pattern that is significantly associated with reaction time and robust to covariates of age, sex, and time from injury. The discovered pattern is specific to cognitive symptoms and does not correlate with ImPACT measures outside of processing speed and reaction time. This article is the first to elucidate the influence of multiple regions jointly on postconcussive reaction time through visualizable FA images.

This work delves deeper in elucidating the link between postconcussive reaction time and brain microstructure than prior studies. ${ }^{29-32}$ Previous studies investigated individual ROIs.
Womack et $\mathrm{al}^{29}$ reported that the posterior corpus callosum is related to peripheral visual reaction time, and Arenth et al ${ }^{30}$ reported that DTI parameters in the corpus callosum relate to impaired reaction time post-TBI. The prior findings, however, lack specificity to cognitive performance because the corpus callosum is commonly involved across mTBI populations. ${ }^{31}$ Our work uncovers microstructural circuitry that is specific to cognitive processing (ie, reaction time and processing speed). Other studies examining frontal ROIs ${ }^{12,32}$ found no consistent pattern between DTI variables on various ROIs and symptom scale scores. Most important, a prior study suggested that cognitive deficit is a diffuse process: the number of DTI lesions positively correlated with poorer cognitive measures. ${ }^{7}$ Our work offers a more complete map of the multiple microstructural lesions that jointly influence reaction time. This study uniquely visualizes the complex, dynamic microstructure opaque to visual interpretation and ROIbased approaches because TBM is generative.

Our work demonstrates a new approach for assessing latent microstructural patterns, especially those that are not discernible 
visually. TBM addresses many shortcomings of traditional methods. Histogram analysis does not provide regionally specific answers, ${ }^{12,33}$ ROI-based techniques do not characterize spatially diffuse processes, and voxel-based analysis is sensitive to a priori structural/functional alignment, ${ }^{12,33,34}$ which produces falsepositives in mTBI cohorts. ${ }^{10}$ Tractography visualizes in vivo pathways, but poorly understood interconnections are precisely the ones most difficult to characterize using tractography. ${ }^{35}$ In contrast, TBM uncovers structure-function circuitry ${ }^{13}$ in an automated manner. Unlike traditional methods, TBM is generative and inverse transformation visualizes latent microstructure opaque to these methodologies. We see multiple white matter tracts dynamically interacting, providing novel insight into biophysical substrates. Our approach is widely applicable to future mTBI studies investigating the link between brain microstructure and clinical symptoms.

The microstructure we discovered is consistent with reaction time neurobiology. First, optic radiations interpret the initial stimulus and contribute to interindividual variability in reaction time. ${ }^{36}$ Next, frontally mediated circuits help process the observed stimulus. In prior studies, damage to the anterior thalamic radiations led to impaired cognitive function ${ }^{37}$ and was associated with reaction time deficits. ${ }^{38}$ Following executive processing, interhemispheric transmission translates attention into a physical response. The corpus callosum mediates interhemispheric transmission and correlates with reaction time in both healthy and cognitively impaired individuals. ${ }^{33,39}$ Finally, the corticospinal tract generates a motor response. ${ }^{40}$

In addition, this study illuminates interaction across multiple white matter tracts. The direction of FA varies among the corpus callosum and visual tracts, corticospinal tracts, and anterior thalamic radiations. Our corpus callosum findings are consistent with prior reports ${ }^{6}$ of increased FA with injury that suggest cytotoxic edema in the semiacute phase. However, other studies describe diffusely decreased FA with persistent cognitive symptoms, including in the corpus callosum. ${ }^{7,41}$ The acute/postacute imaging time period may be responsible for this finding. White matter injury in mTBI is dynamic, demonstrating increased FA early due to cytotoxic edema and decreased FA late, reflecting decreased white matter integrity and axonal loss. ${ }^{42}$ Our finding of mixed FA likely reflects a transitional time period. Some regions may have progressed to axonal loss, while others remain in earlier stages. This mixed response is seen during the semiacute interval. ${ }^{43,44}$ The corpus callosum is the largest fiber tract in the human brain ${ }^{45}$; increased FA may represent residual edema from the immediate into the subacute phase of injury relative to smaller tracts.

There are several limitations of this study. We conducted a retrospective, single-center study. Future prospective, multicenter studies are needed to build on our results. Additionally, our patients ranged from pediatric to adult. White matter myelination may vary in the pediatric population. However, our model remained significant even after accounting for age covariation. ${ }^{46}$ Furthermore, focusing on FA spatial distribution may be advantageous in mixed-age patient populations in which differences in head size may confound traditional voxel-based techniques. Our study included subjects imaged at heterogeneous intervals since traumatic injury, but the statistical model remained robust after correcting for time from injury as a covariate. Furthermore, many patients with $\mathrm{mTBI}$ do not seek medical attention, and there is a possible selection bias toward more seriously injured or symptomatic patients. However, the bias may help direct toward patients who would benefit most from objective assessment. Finally, lack of universally accepted normative data limits quantitative use of DTI metrics in the clinical setting. Future studies will need to establish normative references for quantitative TBM in healthy patients and those with mTBI. This study motivates future investigation into causality between brain microstructure and mTBI symptoms.

\section{CONCLUSIONS}

In the future, 3D TBM may help provide prognostic markers to assess either recovery or progression to chronic stages in prospective longitudinal DTI studies. ${ }^{12,33}$ There is immense potential for 3D-TBM to be a novel diagnostic technology capable of investigating structure-function relationships in many diseases like mTBI that are still considered mysteries.

Disclosures: Gustavo K. Rohde_UNRELATED: Grants/Grants Pending: National Institutes of Health/National Science Foundation, Comments: I currently have 1 National Institutes of Health and National Science Foundation pending grant on developing the underlying technology that will support future studies using transport-based morphometry; Patents (Planned, Pending or Issued): United States Patent Office, Comments: I have 1 patent related to methods for measuring optimal transport between images.

\section{REFERENCES}

1. Vincent AS, Roebuck-Spencer TM, Cernich A, et al. Cognitive changes and dementia risk after traumatic brain injury: implications for aging military personnel. AAlzheimers Dement 2014;10(3 Suppl):S174-87 CrossRef Medline

2. Himanen L, Portin R, Isoniemi $\mathrm{H}$, et al. Cognitive functions in relation to MRI findings 30 years after traumatic brain injury. Brain Inj 2005;19:93-100 Medline

3. Grandhi R, Tavakoli S, Ortega C, et al. A review of chronic pain and cognitive, mood, and motor dysfunction following mild traumatic brain injury: complex, comorbid, and/or overlapping conditions? Brain Sci 2017:7 CrossRef Medline

4. Levin HS, Li X, McCauley SR, et al. Neuropsychological outcome of mTBI: a principal component analysis approach. J Neurotrauma 2013;30:625-32 CrossRef Medline

5. Hellawell DJ, Taylor RT, Pentland B. Cognitive and psychosocial outcome following moderate or severe traumatic brain injury. Brain Inj 1999;13:489-504 Medline

6. Wozniak JR, Krach L, Ward E, et al. Neurocognitive and neuroimaging correlates of pediatric traumatic brain injury: a diffusion tensor imaging (DTI) study. Arch Clin Neuropsychol 2007;22:555-68 Medline

7. Niogi SN, Mukherjee P, Ghajar J, et al. Extent of microstructural white matter injury in postconcussive syndrome correlates with impaired cognitive reaction time: a $3 \mathrm{~T}$ diffusion tensor imaging study of mild traumatic brain injury. AJNR Am J Neuroradiol 2008; 29:967-73 Medline

8. Mac Donald CL, Dikranian K, Bayly P, et al. Diffusion tensor imaging reliably detects experimental traumatic axonal injury and indicates approximate time of injury. J Neurosci 2007;27:11869-76 Medline

9. Davenport ND, Lim KO, Armstrong MT, et al. Diffuse and spatially variable white matter disruptions are associated with blast-related mild traumatic brain injury. Neuroimage 2012;59:2017-24 CrossRef Medline

10. Watts R, Thomas A, Filippi CG, et al. Potholes and molehills: bias in 
the diagnostic performance of diffusion-tensor imaging in concussion. Radiology 2014;272:217-23 CrossRef Medline

11. Ghodadra A, Alhilali L, Fakhran S. Principal component analysis of diffusion tensor images to determine white matter injury patterns underlying postconcussive headache. AJNR Am J Neuroradiol 2016; 37:274-78 CrossRef Medline

12. Niogi SN, Mukherjee P. Diffusion tensor imaging of mild traumatic brain injury. J Head Trauma Rehabil 2010;25:241-55 CrossRef Medline

13. Kundu S, Kolouri S, Erickson KI, et al. Discovery and visualization of structural biomarkers from MRI using transport-based morphometry. Neuroimage 2018;167:256-75 CrossRef Medline

14. Erlanger D, Saliba E, Barth J, et al. Monitoring resolution of postconcussion symptoms in athletes: preliminary results of a webbased neuropsychological test protocol. J Athl Traii 2001;36:280-87 Medline

15. Collie A, Maruff P, Makdissi M, et al. CogSport: reliability and correlation with conventional cognitive tests used in postconcussion medical evaluations. Clin J Sport Med 2003;13:28 -32 Medline

16. Leddy JJ, Kozlowski K, Fung M, et al. Regulatory and autoregulatory physiological dysfunction as a primary characteristic of post concussion syndrome: implications for treatment. NeuroRehabilitation 2007;22:199-205 Medline

17. Leddy JJ, Kozlowski K, Donnelly JP, et al. A preliminary study of subsymptom threshold exercise training for refractory post-concussion syndrome. Clin J Sport Med 2010;20:21-27 CrossRef Medline

18. Department of Defense, Department of Veterans Affairs. VA/DoD Clinical Practice Guideline for Management of Concussion/Mild Traumatic Brain Injury. 2009. https://www.rehab.research.va.gov/ jour/09/46/6/pdf/cpg.pdf. Accessed May 17, 2019

19. Mayers LB, Redick TS. Clinical utility of ImPACT assessment for postconcussion return-to-play counseling: psychometric issues. J Clin Exp Neuropsychol 2012;34:235-42 CrossRef Medline

20. Eckner JT, Kutcher JS, Richardson JK. Effect of concussion on clinically measured reaction time in 9 NCAA division I collegiate athletes: a preliminary study. PM R 2011;3:212-18 CrossRef Medline

21. Lau B, Lovell MR, Collins MW, et al. Neurocognitive and symptom predictors of recovery in high school athletes. Clin J Sport Med 2009; 19:216-21 Medline

22. Behrens TE, Woolrich MW, Jenkinson M, et al. Characterization and propagation of uncertainty in diffusion-weighted MR imaging. Magn Reson Med 2003;50:1077-88 Medline

23. FDT. FMRIB's Diffusion Toolbox. http://fsl.fmrib.ox.ac.uk/fsl/fslwiki/FDT. Updated May 12, 2016. Accessed May 17, 2019

24. Jenkinson M, Beckmann CF, Behrens TE, et al. FSL. Neuroimage 2012;62:782-90 CrossRef Medline

25. FMRIB Software Library, Version 6.0. Analysis Group, FMRIB, Oxford, UK. http://www.fmrib.ox.ac.uk/fsl. Updated April 16, 2019. Accessed March 26, 2016

26. Basu S, Kolouri S, Rohde GK. Detecting and visualizing cell phenotype differences from microscopy images using transport-based morphometry. Proc Natl Acad Sci U S A 2014;111:3448-53 CrossRef Medline

27. Wang W, Slepčev D, Basu S, et al. A linear optimal transportation framework for quantifying and visualizing variations in sets of images. Int J Comput Vis 2013;101:254-69 CrossRef Medline

28. Kolouri S, Tosun AB, Ozolek JA, et al. A continuous linear optimal transport approach for pattern analysis in image datasets. Pattern Recognit 2016;51:453-62 CrossRef Medline
29. Womack KB, Paliotta C, Strain JF, et al. Measurement of peripheral vision reaction time identifies white matter disruption in patients with mild traumatic brain injury. J Neurotrauma 2017;34:1539-45 CrossRef Medline

30. Arenth PM, Russell KC, Scanlon JM, et al. Corpus callosum integrity and neuropsychological performance after traumatic brain injury: a diffusion tensor imaging study. J Head Trauma Rehabil 2014;29: E1-10 CrossRef Medline

31. Aoki Y, Inokuchi R, Gunshin M, et al. Diffusion tensor imaging studies of mild traumatic brain injury: a meta-analysis. J Neurol Neurosurg Psychiatry 2012;83:870-76

32. Levin $\mathrm{H}$, Wilde EA, Troyanskaya $\mathrm{M}$, et al. Diffusion tensor imaging of mild to moderate blast related TBI and its sequelae. $\mathrm{J} \mathrm{Neu-}$ rotrauma 2010;27:683-94 CrossRef Medline

33. Grossman EJ, Inglese M, Bammer R. Mild traumatic brain injury: is diffusion imaging ready for primetime in forensic medicine? Top Magn Reson Imaging 2010;21:379-86 CrossRef Medline

34. Bookstein FL. "Voxel-based morphometry" should not be used with imperfectly registered images. Neuroimage 2001;14:1454-62 Medline

35. Jbabdi S, Johansen-Berg H. Tractography: where do we go from here? Brain Connect 2011;1:169-83 CrossRef Medline

36. Tuch DS, Salat DH, Wisco JJ, et al. Choice reaction time performance correlates with diffusion anisotropy in white matter pathways supporting visuospatial attention. Proc Natl Acad Sci U S A 2005;102:12212-17 Medline

37. Booth $\mathrm{T}$, Bastin ME, Penke L, et al. Brain white matter tract integrity and cognitive abilities in community-dwelling older people: the Lothian Birth Cohort, 1936. Neuropsychology 2013;27:595-607 CrossRef Medline

38. Righart RI, Duering M, Gonik M, et al. Impact of regional cortical and subcortical changes on processing speed in cerebral small vessel disease. Neuroimage Clin 2013;2:854-61 CrossRef Medline

39. Anstey KJ, Mack HA, Christensen H, et al. Corpus callosum size, reaction time speed and variability in mild cognitive disorders and in a normative sample. Neuropsychologia 2007;45:1911-20 Medline

40. Leocani L, Cohen LG, Wassermann EM, et al. Human corticospinal excitability evaluated with transcranial magnetic stimulation during different reaction time paradigms. Brain 2000;123(Pt 6): 1161-73 Medline

41. Salmond CH, Menon DK, Chatfield DA, et al. Diffusion tensor imaging in chronic head injury survivors: correlations with learning and memory indices. Neuroimage 2006;29:117-24 Medline

42. Eierud C, Craddock RC, Fletcher S, et al. Neuroimaging after mild traumatic brain injury: review and meta-analysis. Neuroimage Clin 2014;4:283-94 CrossRef Medline

43. Mayer AR, Ling J, Mannel MV, et al. A prospective diffusion tensor imaging study in mild traumatic brain injury. Neurology 2010;74: 643-50 CrossRef Medline

44. Bazarian JJ, Zhong J, Blyth B, et al. Diffusion tensor imaging detects clinically important axonal damage after mild traumatic brain injury: a pilot study. J Neurotrauma 2007;24:1447-59 Medline

45. Aboitiz F. Brain connections: interhemispheric fiber systems and anatomical brain asymmetries in humans. Biol Res 1992;25:51-61 Medline

46. Eckert MA, Keren NI, Roberts DR, et al. Age-related changes in processing speed: unique contributions of cerebellar and prefrontal cortex. Front Hum Neurosci 2010;4:10 CrossRef Medline 